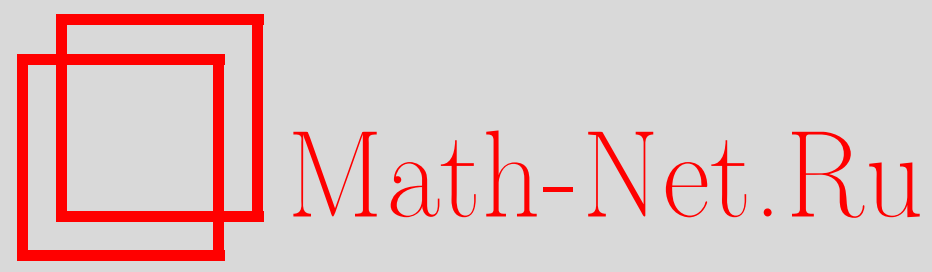

А. А. Махнев, М. С. Нирова, О сильно регулярных графах с $b_{1}<26$, Дискрет. матем., 2013, том 25, выпуск 3, 22-32

DOI: https://doi.org/10.4213/dm1245

Использование Общероссийского математического портала Math-Net.Ru подразумевает, что вы прочитали и согласны с пользовательским соглашением http://www.mathnet.ru/rus/agreement

Параметры загрузки:

IP: 54.237 .206 .68

26 апреля 2023 г., 14:56:22

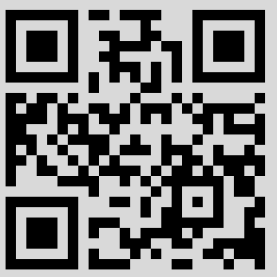




\title{
О сильно регулярных графах с $\mathrm{b}_{1}<26$
}

\author{
() 2013 г. А. А. Махнев, М. С. Нирова
}

Пусть $\Gamma$ - связный реберно регулярный граф с параметрами $(v, k, \lambda)$, $b_{1}=k-\lambda-1$. Хорошо известно, что если $b_{1}=1$, то $\Gamma-$ многоугольник или полный многодольный граф с долями порядка 2. Ранее были классифицированы графы с $b_{1} \leqslant 4$. Изучение графов даже в случае $b_{1}=5$ идет с большим трудом. Однако для сильно регулярных графов ситуация гораздо проще. В данной работе классифицированы сильно регулярные графы с $b_{1}<26$.

Работа выполнена при поддержке Российско-Словенского проекта 2012-2013 гг., программы отделения математических наук РАН (проект 12-Т-1-1003) и программ совместных исследований УрО РАН с СО РАН (проект 12-С-1-1018) и с НАН Беларуси (проект 12-С-1-1009).

Мы рассматриваем неориентированные графы без петель и кратных ребер. Для вершины $a$ графа $\Gamma$ через $[a]$ обозначается окрестность $a$, т.е. подграф графа Г, индуцированный множеством вершин, смежных с $a$. Для вершин $a, b$ графа $Г$ через $d(a, b)$ обозначим расстояние между $a, b$ в графе Г. Под собственными значениями графа понимаются собственные значения его матрицы смежности. Пусть $\mathcal{F}$ - семейство графов. Граф Г называется локально $\mathcal{F}$ графом, если $[a] \in \mathcal{F}$ для любой вершины $a \in \Gamma$.

Граф Г называется регулярным графом степени $k$, если $[a]$ содержит точно $k$ вершин для любой вершины $a$ из Г. Граф Г называется реберно регулярным графом c параметрами $(v, k, \lambda)$, если Г содержит $v$ вершин, является регулярным степени $k$, и каждое ребро графа Г лежит точно в $\lambda$ треугольниках. Граф Г называется силъно регулярным с параметрами $(v, k, \lambda, \mu)$, если $Г$ реберно регулярен с соответствующими параметрами и подграф $[a] \cap[b]$ содержит точно $\mu$ вершин для любых двух несмежных вершин $a, b$. Подграф $[a] \cap[b]$ назовем $\lambda$-подграфом ( $\mu$-подграфом), если $d(a, b)=1$ (если $d(a, b)=2)$.

Через $K_{m_{1}, \ldots, m_{n}}$ обозначим полный $n$-дольный граф, с долями порядков $m_{1}, \ldots, m_{n}$. Если $m_{1}=\ldots=m_{n}=m$, то соответствующий граф обозначается через $K_{n \times m}$. Граф $K_{1, m}$ называется $m$-лапой. Треугольным графом $T(m)$ называется граф с множеством неупорядоченных пар из $X$ в качестве вершин, $|X|=m$ и пары $\{a, b\},\{c, d\}$ смежны тогда и только тогда, когда они имеют единственный общий элемент. Граф на множестве вершин $X \times Y$ называется $m \times n$ решеткой, если $|X|=m,|Y|=n$ и вершины $\left(x_{1}, y_{1}\right),\left(x_{2}, y_{2}\right)$ смежны тогда и только тогда, когда $x_{1}=x_{2}$ или $y_{1}=y_{2}$. Графом Джсосона $J(n, m)$ называется граф, вершинами которого являются $m$-элементные подмножества данного $n$-элементного множества, причем две вершины $a, b$ смежны, только если $|a \cap b|=m-1$. Граф Пэли $P(q)$ в качестве вершин имеет элементы поля $F_{q}, q \equiv 1(\bmod 4)$, и две вершины $a, b$ смежны, 
только если $b-a$ является ненулевым квадратом в $F_{q}$. Граф Петерсена - это дополнительный граф для треугольного графа $T(5)$ (он имеет параметры $(10,3,0,1)$ ). Граф Клебша (Шлефли) - это единственный сильно регулярный граф с параметрами $(16,10,6,6)$ (с параметрами $(27,16,10,8))$. Граф Шрикханде - это единственный сильно регулярный локально шестиугольный граф с параметрами $(16,6,2,2)$. Имеется точно 3 сильно регулярных графа, имеющих параметры графа $T(8)$, но не изоморфных $T(8)$. Эти графы называются графами Чанга. Графом Хофмана-Синглтона называется единственный сильно регулярный граф с параметрами $(50,7,0,1)$. Графом Хигмена-Cимса называется единственный сильно регулярный граф с параметрами $(100,22,0,6)$.

Частичной геометрией $p G_{\alpha}(s, t)$ называется система инцидентности, состоящая из точек и прямых, в которой каждая прямая содержит $s+1$ точку, каждая точка лежит на $t+1$ прямой (две прямые пересекаются не более, чем по одной точке) и для любой точки $a$, не лежащей на прямой $L$, найдется точно $\alpha$ прямых, проходящих через $а$ и пересекающих $L$. Если $\alpha=1$, то геометрия называется обобщенным четырехугольником и обозначается $G Q(s, t)$. Если $\alpha=t$, то геометрия называется сетъю. Точечным графом частичной геометрии называется граф, вершинами которого являются точки геометрии, и две различные вершины смежны, если они лежат на одной прямой. Легко понять, что точечный граф частичной геометрии $p G_{\alpha}(s, t)$ сильно регулярен с параметрами: $v=(s+1)(1+s t / \alpha), k=s(t+1), \lambda=(s-1)+(\alpha-1) t$, $\mu=\alpha(t+1)$. Любой сильно регулярный граф с такими параметрами для некоторых $\alpha, s, t$ называется псевдогеометрическим графом для $p G_{\alpha}(s, t)$. Заметим, что если $\Gamma$ является псевдогеометрическим графом для $p G_{\alpha}(s, t), \alpha$ делит $s t$ и $\alpha<s$, то граф $\bar{\Gamma}$ является псевдогеометрическим графом для $p G_{t(s-\alpha) / \alpha}(s t / \alpha, s-\alpha)$.

Сильно регулярные графы с собственным значением -2 были классифицированы Зейделем (теорема 3.12.4 [1]). Любой зейделев граф - это либо полный многодольный граф $K_{r \times 2}$, либо решетчатый или треугольный граф, либо один из графов Шрикханде, Чанга, Петерсена, Клебша или Шлефли.

Если вершины $u, w$ находятся на расстоянии $i$ в $\Gamma$, то через $b_{i}(u, w)$ (через $\left.c_{i}(u, w)\right)$ обозначим число вершин в пересечении $\Gamma_{i+1}(u)$ (пересечении $\left.\Gamma_{i-1}(u)\right)$ с $[w]$. Заметим, что в реберно регулярном графе с параметрами $(v, k, \lambda)$ значение $b_{1}=b_{1}(u, w)$ не зависит от выбора ребра $\{u, w\}$ и равно $k-\lambda-1$.

В следствии 1.1.6 из [1] доказано, что если $\Gamma-$ связный реберно регулярный граф с $b_{1}=1$, то $\Gamma$ - многоугольник или полный многодольный граф $K_{n \times 2}$. Реберно регулярные графы с $2 \leqslant b_{1} \leqslant 5$ изучались в работах [2-3]. Изучение реберно регулярных графов даже в случае $b_{1}=5$ идет с большим трудом. Однако для сильно регулярных графов ситуация гораздо проще. В данной работе классифицированы сильно регулярные графы с $b_{1}<26$.

Теорема 1. Пусть $Г-$ силъно регулярный граф с $0<b_{1}<26$. Тогда Г является одним из следующих графов:

(1) граф с параметрами $\left(4 b_{1}+1,2 b_{1}, b_{1}-1, b_{1}\right), b_{1} \notin\{5,8,14,17,19,23\}$, или полный многодольный граф $K_{r \times\left(b_{1}+1\right)}$;

(2) зейделев граф или его дополнение;

(3) псевдогеометрический граф для $G Q(s, t), \quad\{s, t\}$ $\{\{2,2\},\{2,4\},\{3,3\},\{3,5\},\{3,6\},\{4,4\},\{4,6\}\},\{5,5\}\}$, или его дополнение;

(4) псевдогеометрический граф для сети $p G_{t}(s, t)$, где либо $t=2, s=3,4,5, \ldots, 13$, либо $t=3, s=4,5, \ldots, 10$, либо $t=4, s=6,7,8,9$, либо $t=5, s=8,9$, либо $t=s-2$, $s=7,8,9,10$, либо $t=12, s=13$; 
(5) псевдогеометрический граф для $p G_{\alpha}(5+\alpha, 4), \alpha \in\{1, \ldots, 6,15,20,30,60\}$, $p G_{2}(4, t) \quad(t=3,7), p G_{2}(5,5), p G_{3}(s, 2) \quad(s=5,6,8,9,12), p G_{3}(5,7), p G_{4}(7,2)$, $p G_{4}(7,3), p G_{4}(8,3), p G_{4}(11,3), p G_{5}(9,3), p G_{5}(14,2), p G_{6}(8,5), p G_{6}(15,2), p G_{8}(15,2)$, $p G_{9}(18,2), p G_{9}(15,3), p G_{10}(15,3), p G_{14}(20,3), p G_{14}(21,3), p G_{15}(24,2), p G_{15}(19,3)$ или $p G_{20}(24,3)$;

(6) дополнительный граф к графу из пункта (5);

(7) граф с параметрами $(50,7,0,1),(56,10,0,2),(77,16,0,4),(81,20,1,6)$, $(82,36,15,16),(85,30,11,10),(85,14,3,2),(99,14,1,2),(100,33,14,9),(100,22,0,6)$, $(120,77,52,44), \quad(126,25,8,4), \quad(136,30,8,6), \quad(155,42,17,9), \quad(162,23,4,3)$, $(196,39,14,6),(243,22,1,2),(300,26,4,2),(400,21,2,1)$ или его дополнение.

Сначала приведем некоторые вспомогательные результаты.

Лемма 1. Пусть Г - сильно регулярный граф, имеющий параметры $(v, k, \lambda, \mu)$. Тогда либо $k=2 \mu, \lambda=\mu-1$ (так называемый половинный случай) $и$ я является суммой квадратов двух целых чисел, либо неглавные собственные значения $n-$ $m,-m$ графа $\Gamma$ - целые числа, где $n^{2}=(\lambda-\mu)^{2}+4(k-\mu), n-\lambda+\mu=2 m u$ кратность собственного значения $n-m$ равна $\frac{k(m-1)(k+m)}{\mu n}$. Далее, если $m-$ целое число, большее 1 , то $m-1$ делит $k-\lambda-1$ u

$$
\mu=\lambda+2+(m-1)-\frac{k-\lambda-1}{m-1}, n=m-1+\frac{k-\lambda-1}{m-1} .
$$

Доказательство. Это лемма 3.1 из [4].

Лемма 2. Пусть Г - силъно регулярный граф, имеющий целочисленнье собственные значения $k, n-m,-m, u b_{1}=k-\lambda-1$. Тогда

(1) $b_{1}=(n-m+1)(m-1)$;

(2) если $m-1=b_{1}$, то Г является полным многодольным графом $K_{r \times\left(b_{1}+1\right)}$;

(3) если $b_{1}$ - простое число, то Г является полным многодольным графом $K_{r \times\left(b_{1}+1\right)}$ или зейделевым графом;

(4) если $b_{1}=2(m-1)$, то Г является дополнительным к зейделеву графу.

Доказательство. Напомним, что $\lambda-\mu=(n-m)-m$ и $k-\mu=m(n-m)$. Поэтому $b_{1}=m(n-m)-(n-m)+m-1=(m-1)(n-m+1)$. Утверждение (1) доказано.

Если $m-1=b_{1}$, то по утверждению (1) получим $n-m=0$ и Г является полным многодольным графом $K_{r \times\left(b_{1}+1\right)}$. Утверждение $(2)$ доказано.

Если $b_{1}-$ простое число, то $m-1=b_{1}$ или 1 . В первом случае $Г$ является полным многодольным графом $K_{r \times\left(b_{1}+1\right)}$, а во втором случае - зейделевым графом. Утверждение (3) доказано.

Если $b_{1}=2(m-1)$, то $n-m=1$ и $Г$ является дополнительным к зейделеву графу.

Лемма 3. Пусть $Г-$ сильно регулярный граф. Если $k=2 \mu, \lambda=\mu-1 u b_{1}<26$, mo $b_{1} \neq 5,8,14,17,19,23$.

Доказательство. Если $v=4 \mu+1$ является степенью простого числа, то граф Пэли на $v$ вершинах имеет параметры $(v, 2 \mu, \mu-1, \mu)$. Если $b_{1} \leqslant 23$, то $v=4 b_{1}+1$ не является степенью простого числа для $b_{1}=5,8,11,14,16,17,19,21$ или 23. Но в случае $b_{1}=11$ число $v=45$ равно $36+9$, в случае $b_{1}=16$ число $v=65$ равно $49+16$, а в случае $b_{1}=21$ число $v=85$ равно $81+4$. 
Лемма 4. Пусть Г- сильно регулярный граф с $0<b_{1}<26$. Тогда выполняются следующие утверждения:

(1) если Г - граф Зейделя, то Г является полным многодольным графом $K_{r \times 2}$, $n \times n$ решеткой, $n \leqslant 26$, треугольным графом $T(n), n \leqslant 28$, графом Петерсена, Клебша, Шлефли, Шрикханде или одним из трех графов Чанга;

(2) если Г - дополнительный граф $к$ графу Зейделя, то Г является дополнительным графом $\kappa n \times n$ решетке, $n \leqslant 14$, треугольному графу $T(n), n \leqslant 16$, графу Петерсена, Клебша, Шлефли, Шрикханде или одному из трех графов Чанга.

Доказательство. Если Г является $n \times n$ решеткой, то $k=2(n-1), \lambda=n-2$ и $b_{1}=n-1$, поэтому $n \leqslant 26$. Если Г является треугольным графом $T(n)$, то $k=$ $2(n-2), \lambda=n-2$ и $b_{1}=n-3$, поэтому $n \leqslant 28$. Если Г является графом Петерсена, Клебша, Шлефли, Шрикханде или одним из трех графов Чанга, то $b_{1}=2,3,5,3$ или 5 соответственно. Утверждение (1) доказано.

Если $\Gamma$ - дополнительный граф к сильно регулярному графу $\bar{\Gamma}$ с параметрами $(\bar{v}, \bar{k}, \bar{\lambda}, \bar{\mu})$, то $b_{1}=\bar{k}-\bar{\mu}$. Если $\bar{\Gamma}$ является $n \times n$ решеткой, то $b_{1}=2 n-4$, поэтому $n \leqslant 14$. Если $\bar{\Gamma}$ является треугольным графом $T(n)$, то $b_{1}=2 n-8$, поэтому $n \leqslant 16$. Если $\bar{\Gamma}$ является графом Петерсена, Клебша, Шлефли, Шрикханде или одним из трех графов Чанга, то $b_{1}=2,4,8,4$ или 8 соответственно. Лемма доказана.

Пусть до конца работы $Г$ является сильно регулярным графом с параметрами $(v, k, \lambda, \mu)$, не являющимся графом в половинном случае, имеющим собственное значение $-m, 1<m-1<b_{1} / 2$ и $b_{1} \leqslant 25$. Заметим, что если Г является псевдогеометрическим графом для $p G_{\alpha}(s, t)$, то $b_{1}=t(s-\alpha+1)$.

Лемма 5. Если $b_{1}=6$, то либо Г является графом Хофмана-Синглтона или его дополнением, либо Г является графом с параметрами $(26,10,3,4)$ или его дополнением.

Доказательство. По предположению $m-1=2$ и по лемме 2 получим $n-m+1=3$. Но в этом случае граф $\bar{\Gamma}$ также имеет собственные значения 2 и -3 , поэтому $\bar{b}_{1}=6$.

Теперь $k=6+\mu, n=5$, по прямоугольному соотношению $\mu$ делит 36 , а по условию целочисленности $5 \mu$ делит $2(6+\mu)(9+\mu)$. Поэтому $\mu$ сравнимо с \pm 1 по модулю 5 . Если $\mu=1$, то Г имеет параметры $(50,7,0,1)$ и является графом ХофманаСинглтона.

Если $\mu=4$, то Г имеет параметры $(26,10,3,4)$. Если $\mu=6$, то Г является графом в половинном случае. Если $\mu=9$, то Г имеет параметры $(26,15,8,9)$ и является дополнительным графом к графу с параметрами $(26,10,3,4)$. Если же $\mu=36$, то $\Gamma$ является дополнительным графом к графу Хофмана-Синглтона. Лемма доказана.

Заметим, что граф с параметрами $(26,15,8,9)$ является псевдогеометрическим графом для $p G_{3}(5,2)$.

Лемма 6. Если $b_{1}=8$, то выполняется одно из следующих утверждений:

(1) Г является псевдогеометрическим графом для $G Q(4,2), p G_{2}(5,2), p G_{3}(6,2)$ или $\mathrm{pG}_{4}(7,2)$;

(2) Г является дополнительным графом для псевдогеометрического графа обобщенного четырехугольника $G Q(3,3)$ или для графа Гевиртца. 
Доказательство. По предположению $m-1=2$ и по лемме 2 получим $n-m+1=4$. В этом случае граф $\bar{\Gamma}$ имеет собственные значения 2 и -4 , поэтому $\bar{b}_{1}=9$.

Теперь $k=9+\mu, \lambda=\mu, n=6$, по прямоугольному соотношению $\mu$ делит 72 , а по условию целочисленности $6 \mu$ делит $2(9+\mu)(12+\mu)$. Поэтому $\mu$ делится на 3 и делит 36 . Если $\mu=3$, то Г имеет параметры $(45,12,3,3)$ и является псевдогеометрическим графом для обобщенного четырехугольника $G Q(4,2)$.

Если $\mu=6$, то $Г$ имеет параметры $(36,15,6,6)$ и является псевдогеометрическим графом для сети $p G_{2}(5,2)$. Если $\mu=9$, то $Г$ имеет параметры $(35,18,9,9)$ и является псевдогеометрическим графом для $p G_{3}(6,2)$. Заметим, что в этом случае дополнительный граф для Г является псевдогеометрическим графом для $p G_{2}(4,3)$.

Если $\mu=12$, то $Г$ имеет параметры $(36,21,12,12)$ и является псевдогеометрическим графом для $p G_{4}(7,2)$. Если $\mu=18$, то $\Gamma$ имеет параметры $(40,27,18,18)$ и является дополнительным графом для псевдогеометрического графа обобщенного четырехугольника $G Q(3,3)$. Если же $\mu=36$, то Г имеет параметры $(56,45,36,36)$ и является дополнительным графом для графа Гевиртца с параметрами $(56,10,0,2)$.

Лемма 7. Если $b_{1}=9$, то Г является дополнительным графом для одного из графбов в заключении леммы 6.

Доказательство. По предположению $m-1=3$ и по лемме 2 получим $n-m+1=3$. В этом случае граф $\bar{\Gamma}$ имеет собственные значения 3 и -3 , поэтому $\bar{b}_{1}=8$ и $\bar{m}=3$.

Лемма 8. Если $b_{1}=10$, то Г имеет параметры $(85,14,3,2)$ или $(49,18,7,6)$.

Доказательство. По предположению $m-1=2$ и по лемме 2 получим $n-m+1=5$. В этом случае граф $\bar{\Gamma}$ имеет собственные значения 2 и -5, поэтому $\bar{b}_{1}=12$.

Теперь $k=12+\mu, \lambda=\mu+1, n=7$, по прямоугольному соотношению $\mu$ делит 120 , а по условию целочисленности $7 \mu$ делит $2(12+\mu)(15+\mu)$. Поэтому $\mu$ сравнимо с 2 или -1 по модулю 7. Если $\mu=2$, то Г имеет параметры $(85,14,3,2)$.

Если $\mu=6$, то Г имеет параметры $(49,18,7,6)$ и является псевдогеометрическим графом для сети $p G_{2}(6,2)$. Если $\mu=20$, то Г имеет параметры $(49,32,21,20)$ и не существует по [5]. Если $\mu=30$, то Г имеет параметры $(57,42,31,30)$. Но дополнительный граф для этого графа имеет параметры $(57,14,1,4)$ и не существует по [6]. Если же $\mu=120$, то Г имеет параметры $(144,132,121,120)$. Противоречие с тем, что $\bar{\lambda}=v-2 k-2+\mu=-2$.

Лемма 9. Если $b_{1}=12$, то выполняется одно из следующих утверждений:

(1) Г является дополнительным графом для одного из графов в заключении леммы 8;

(2) либо Г имеет параметры $(99,14,1,2)$ или $(50,21,8,9)$, либо Г является дополнительным графом для одного из графов с указанными параметрами;

(3) Г либо имеет параметры $(69,20,7,5)$, либо является псевдогеометрическим грабом для $p G_{2}(7,2)$ или $p G_{3}(8,2)$;

(4) $Г$ является дополнительным графом либо для псевдогеометрического графа обобщенного четырехугольника $G Q(3,5)$, либо для графа с параметрами $(77,16,0,4)$.

Доказательство. По предположению $m-1=2,3$ или 4 . Если $m-1=4$, то граф $\bar{\Gamma}$ имеет собственные значения 4 и -3 , поэтому $\bar{b}_{1}=10$ и Г является дополнительным графом для одного из графов в заключении леммы 8. 
Если $m-1=3$, то граф Г имеет собственные значения 3 и -4 . В этом случае граф $\bar{\Gamma}$ также имеет собственные значения 3 и -4, поэтому $\bar{b}_{1}=12$.

Теперь $k=12+\mu, \lambda=\mu-1, n=7$, по прямоугольному соотношению $\mu$ делит 144 , а по условию целочисленности $7 \mu$ делит $3(12+\mu)(16+\mu)$. Поэтому $\mu$ сравнимо с \pm 2 по модулю 7 . Если $\mu=2$, то Г имеет параметры $(99,14,1,2)$. Если $\mu=9$, то $\Gamma$ имеет параметры $(50,21,8,9)$.

Если $\mu=12$, то Г является графом в половинном случае. Если $\mu=16$, то Г имеет параметры $(50,28,15,16)$ и является псевдогеометрическим графом для $p G_{4}(7,3)$. Если же $\mu=72$, то Г имеет параметры $(99,84,71,72)$.

Если $m-1=2$, то граф Г имеет собственные значения 5 и -3 . В этом случае граф $\bar{\Gamma}$ имеет собственные значения 2 и -6, поэтому $\bar{b}_{1}=15$.

Теперь $k=15+\mu, \lambda=\mu+2, n=8$, по прямоугольному соотношению $\mu$ делит 180 , а по условию целочисленности $8 \mu$ делит $2(15+\mu)(18+\mu)$. Поэтому $\mu$ сравнимо с 1 по модулю 4 или с -2 по модулю 8. Если $\mu=1$, то $Г$ имеет параметры $(209,16,3,1)$. Противоречие с тем, что число 5 -клик в $Г$ равно $209 \cdot 4 / 5$. Если $\mu=5$, то $Г$ имеет параметры $(69,20,7,5)$. Если $\mu=6$, то $\Gamma$ имеет параметры $(64,21,8,6)$ и является псевдогеометрическим графом для сети $p G_{2}(7,2)$. Если $\mu=9$, то Г имеет параметры $(57,24,11,9)$ и является псевдогеометрическим графом для $p G_{3}(8,2)$.

Если $\mu=30$, то Г имеет параметры $(64,45,32,30)$ и является дополнительным графом для псевдогеометрического графа обобщенного четырехугольника $G Q(3,5)$. Если $\mu=45$, то $Г$ имеет параметры $(77,60,47,45)$ и является дополнительным графом для графа с параметрами $(77,16,0,4)$.

Лемма 10. Если $b_{1}=14$, то либо Г является псевдогеометрическим графом для $p G_{2}(8,2)$ или $p G_{3}(9,2)$, либо $Г-$-ополнительный граф для графа с параметрами $(81,20,1,6)$.

Доказательство. По предположению $m-1=2$ и $n-m+1=7$, граф $\bar{\Gamma}$ имеет собственные значения 2 и -7 , поэтому $\bar{b}_{1}=18$.

Теперь $k=18+\mu, \lambda=\mu+3, n=9$, по прямоугольному соотношению $\mu$ делит $18 \cdot 14$, а по условию целочисленности $9 \mu$ делит $2(18+\mu)(21+\mu)$. Поэтому $\mu$ сравнимо с 0 или -3 по модулю 9 . Если $\mu=6$, то $\Gamma$ имеет параметры $(81,24,9,6)$ и является псевдогеометрическим графом для сети $p G_{2}(8,2)$. Если $\mu=9$, то Г имеет параметры $(70,27,12,9)$ и является псевдогеометрическим графом для $p G_{3}(9,2)$.

Если $\mu=36$, то Г имеет параметры $(76,54,39,36)$. В этом случае дополнительный граф для $Г$ является псевдогеометрическим графом для $G Q(3,6)$ и по [7] не существует. Если $\mu=42$, то $\Gamma$ имеет параметры $(81,60,45,42)$ и является дополнительным графом для графа с параметрами $(81,20,1,6)$. Если же $\mu=63$, то Г имеет параметры $(100,81,66,63)$ и $\bar{\lambda}=-1$.

Лемма 11. Если $b_{1}=15$, то выполняется одно из следующих утверждений:

(1) либо Г является псевдогеометрическим графом для $G Q(3,5)$ или единственным сильно регулярным графом с параметрами $(77,16,0,4)$, либо Г является дополнительным графом для графа с параметрами $(69,20,7,5),(64,21,8,6)$ или $(57,24,11,9)$;

(2) Г является псевдогеометрическим графом для $G Q(5,3), p G_{3}(7,3), p G_{4}(8,3)$, $p G_{5}(9,3), p G_{12}(16,3), p G_{15}(19,3)$ или $p G_{20}(24,3)$.

Доказательство. По предположению $m-1=3$ или 5 . Если $m-1=5$, то $n-m+1=$ 3 , поэтому граф $\bar{\Gamma}$ имеет собственные значения 5 и -3 , поэтому $\bar{b}_{1}=12$ и $\bar{m}-1=2$. В этом случае по лемме 9 выполняется утверждение (1). 
Если $m-1=3$, то $n-m+1=5$, поэтому граф $\bar{\Gamma}$ имеет собственные значения 3 и -5. Теперь $k=16+\mu, \lambda=\mu, n=8$, по прямоугольному соотношению $\mu$ делит $16 \cdot 15$, а по условию целочисленности $8 \mu$ делит $3(16+\mu)(20+\mu)$. Поэтому $\mu$ сравнимо с 4 по модулю 8 или делится на 16. Если $\mu=4$, то Г имеет параметры $(96,20,4,4)$ и является псевдогеометрическим графом для $G Q(5,3)$. Если $\mu=12$, то Г имеет параметры $(64,28,12,12)$ и является псевдогеометрическим графом для $p G_{3}(7,3)$.

Если $\mu=16$, то Г имеет параметры $(63,32,16,16)$ и является псевдогеометрическим графом для $p G_{4}(8,3)$. Если $\mu=20$, то $Г$ имеет параметры $(64,36,20,20)$ и является псевдогеометрическим графом для $p G_{5}(9,3)$. Если $\mu=48$, то Г имеет параметры $(85,64,48,48)$ и является псевдогеометрическим графом для $p G_{12}(16,3)$. Если $\mu=60$, то Г имеет параметры $(96,76,60,60)$ и является псевдогеометрическим графом для $p G_{15}(19,3)$. Если $\mu=80$, то Г имеет параметры $(115,96,80,80)$ и является псевдогеометрическим графом для $p G_{20}(24,3)$. является дополнительным графом для графа с параметрами $(115,18,1,3)$. Если же $\mu=240$, то Г имеет параметры $(273,256,240,240)$ и $\bar{\lambda}=-1$. Лемма доказана.

Заметим, что граф с параметрами $(69,48,32,36)$ является псевдогеометрическим для $p G_{6}(8,5)$, а псевдогеометрический граф для $p G_{20}(24,3)$ является дополнительным графом для графа с параметрами $(115,18,1,3)$.

Лемма 12. Если $b_{1}=16$, то выполняется одно из следующих утверждений:

(1) Г имеет параметры $(126,25,8,4)$ частного графа Дюнонсона $J(10,5)$;

(2) Г является либо псевдогеометрическим графом для $p G_{2}(9,2)$ или $p G_{8}(15,2)$, либо дополнительным графом $\kappa$ псевдогеометрическому графу для $p G_{2}(4,7)$ или $p G_{3}(5,7)$, либо дополнительным графом к графу Хигмена-Симса;

(3) Г является дополнительным графом для графа из пункта (2) леммы 11.

Доказательство. По предположению $m-1=2$ или 4 . Если $m-1=2$, то $n-m+1=8$, поэтому граф $\bar{\Gamma}$ имеет собственные значения 2 и -8 , поэтому $\bar{b}_{1}=21$. Теперь $k=21+\mu, \lambda=4+\mu, n=10$, по прямоугольному соотношению $\mu$ делит $21 \cdot 16$, а по условию целочисленности $10 \mu$ делит $2(21+\mu)(24+\mu)$. Поэтому $\mu$ делит 168 и $\mu$ сравнимо с \pm 1 по модулю 5. Если $\mu=1$, то Г имеет параметры $(375,22,6,1)$ и окрестность вершины в $Г$ не может быть объединением 7 -клик. Если $\mu=4$, то $Г$ имеет параметры $(126,25,8,4)$. Если $\mu=6$, то $Г$ имеет параметры $(81,27,10,6)$ и является псевдогеометрическим графом для $p G_{2}(9,2)$. Если $\mu=14$, то Г имеет параметры $(76,35,18,14)$ и является дополнительным графом к псевдогеометрическому графу для $p G_{3}(5,7)$. Если $\mu=21$, то Г имеет параметры $(75,42,25,21)$ и является дополнительным графом к псевдогеометрическому графу для $p G_{2}(4,7)$. Если $\mu=24$, то Г имеет параметры $(76,45,28,24)$ и является псевдогеометрическим графом для $p G_{8}(15,2)$. Если $\mu=56$, то Г имеет параметры $(100,77,60,56)$ и является дополнительным графом к графу Хигмена-Симса. Наконец, если $\mu=84$, то Г имеет параметры $(126,105,88,84)$ и в дополнительном графе получим $\bar{\lambda}=-2$.

Если $m-1=4$, то $n-m+1=4$, поэтому граф $\bar{\Gamma}$ имеет собственные значения 4 и -4. В этом случае $\bar{b}_{1}=15$ и Г является дополнительным графом для графа из пункта (2) леммы 11.

Лемма 13. Если $b_{1}=18$, то выполняется одно из следующих утверждений:

(1) Г имеет параметры $(100,33,14,9)$;

(2) Г имеет параметры $(400,21,2,1),(162,23,4,3),(81,32,13,12),(81,50,31,30)$ или $(85,30,11,10)$; 
(3) Г является псевдогеометрическим графом для $G Q(6,3)$ или для $p G_{10}(15,3)$;

(4) Г либо имеет параметры $(81,20,1,6)$, либо является псевдогеометрическим графом для $p G_{6}(8,6)$ или $p G_{4}(6,6)$.

Доказательство. По предположению $m-1=2,3$ или 6 . Если $m-1=2$, то $n-m+1=$ 9 , поэтому граф $\bar{\Gamma}$ имеет собственные значения 2 и -9 , поэтому $\bar{b}_{1}=24$. Теперь $k=24+\mu, \lambda=5+\mu, n=11$, по прямоугольному соотношению $\mu$ делит $24 \cdot 18$, а по условию целочисленности $11 \mu$ делит $2(24+\mu)(27+\mu)$. Поэтому $\mu$ делит $24 \cdot 54$ и 11 делит $(\mu+2)(\mu+5)$. Если $\mu=6$, то $Г$ имеет параметры $(121,30,11,6)$ и $Г$ является псевдогеометрическим графом для $p G_{2}(10,2)$. Если $\mu=9$, то Г имеет параметры $(100,33,14,9)$. Если $\mu=72$, то $Г$ имеет параметры $(121,96,77,72)$ и $\bar{\lambda}=-1$. Если $\mu=108$, то $\Gamma$ имеет параметры $(155,132,113,108)$ и $\bar{\lambda}=-3$.

Если $m-1=3$, то $n-m+1=6$, поэтому граф $\bar{\Gamma}$ имеет собственные значения 3 и -6. В этом случае $\bar{b}_{1}=20$. Теперь $k=20+\mu, \lambda=1+\mu, n=9$, по прямоугольному соотношению $\mu$ делит $20 \cdot 18$, а по условию целочисленности $9 \mu$ делит $3(20+\mu)(24+\mu)$. Поэтому $\mu$ делит $24 \cdot 60$ и 3 делит $\mu(\mu+2)$. Если $\mu=1$, то Г имеет параметры $(400,21,2,1)$. Если $\mu$ делится на 3 , то 9 делит $24+\mu$, поэтому либо $\mu=3$ и Г имеет параметры $(162,23,4,3)$, либо $\mu=12$ и Г имеет параметры $(81,32,13,12)$, либо $\mu=30$ и Г имеет параметры $(81,50,31,30)$. Если $\mu=4$, то Г имеет параметры $(133,24,5,4)$ и является псевдогеометрическим графом для $G Q(6,3)$. Если $\mu=10$, то Г имеет параметры $(85,30,11,10)$. Если $\mu=40$, то Г имеет параметры $(88,60,41,40)$ и является псевдогеометрическим графом для $p G_{10}(15,3)$. Если же $\mu \geqslant 90$, то $\bar{\lambda}<0$.

Если $m-1=6$, то $n-m+1=3$, граф $\bar{\Gamma}$ имеет собственные значения 6 и 3 , поэтому $\bar{b}_{1}=14$. По лемме 10 граф $\Gamma$ либо имеет параметры $(81,20,1,6)$, либо является псевдогеометрическим графом для $p G_{6}(8,6)$ или $p G_{4}(6,6)$.

Лемма 14. Если $b_{1}=20$, то выполняется одно из следующих утверждений:

(1) $Г$ является псевдогеометрическим графом для $p G_{3}(12,2), p G_{5}(14,2)$, $p G_{6}(15,2), p G_{9}(18,2)$ или для $p G_{15}(24,2)$;

(2) Г имеет параметры $(243,22,1,2),(82,36,15,16)$ или $(82,45,24,25)$;

(3) Г является дополнительным графом для графа из пунктов (2-3) заключения леммы 13.

Доказательство. По предположению $m-1=2,4$ или 5 . Если $m-1=2$, то $n-m+1=$ 10 , поэтому граф $\bar{\Gamma}$ имеет собственные значения 2 и -10, поэтому $\bar{b}_{1}=27$. Теперь $k=27+\mu, \lambda=6+\mu, n=12$, по прямоугольному соотношению $\mu$ делит $27 \cdot 20$, а по условию целочисленности $12 \mu$ делит $2(27+\mu)(30+\mu)$. Поэтому $\mu$ делит $27 \cdot 10$ и 6 делит $(\mu+3) \mu$. Если $\mu=3$, то $\Gamma$ имеет параметры $(231,30,9,3)$ и $Г$ является псевдогеометрическим графом для $G Q(10,2)$, противоречие. Если $\mu=6$, то Г имеет параметры $(144,33,12,6)$ и является псевдогеометрическим графом для $p G_{2}(11,2)$. Если $\mu=9$, то $Г$ имеет параметры $(117,36,15,9)$ и является псевдогеометрическим графом для $p G_{3}(12,2)$. Если $\mu=15$, то $Г$ имеет параметры $(99,42,21,15)$ и является псевдогеометрическим графом для $p G_{5}(14,2)$. Если $\mu=18$, то $Г$ имеет параметры $(96,45,24,18)$ и является псевдогеометрическим графом для $p G_{6}(15,2)$. Если $\mu=27$, то Г имеет параметры $(95,54,33,27)$ и является псевдогеометрическим графом для $p G_{9}(18,2)$. Если $\mu=30$, то $\Gamma$ имеет параметры $(96,57,36,30)$ и не существует (см. домашнюю стр. А. Броувер). Если $\mu=45$, то $Г$ имеет параметры $(105,72,51,45)$ и является псевдогеометрическим графом для $p G_{1} 5(24,2)$.

Если $m-1=4$, то $n-m+1=5$, поэтому граф $\bar{\Gamma}$ имеет собственные значения 4 и -5. В этом случае $\bar{b}_{1}=20$. Теперь $k=20+\mu, \lambda=\mu-1, n=9$, по прямоугольному 
соотношению $\mu$ делит $20 \cdot 20$, а по условию целочисленности $9 \mu$ делит $4(20+\mu)(25+\mu)$. Поэтому 9 делит $(\mu-2)(\mu+2)$. Если $\mu=2$, то $\Gamma$ имеет параметры $(243,22,1,2)$. Если $\mu=16$, то Г имеет параметры $(82,36,15,16)$. Если $\mu=20$, то $\Gamma$ - граф в половинном случае. Если $\mu=25$, то Г имеет параметры $(82,45,24,25)$.

Если $m-1=5$, то $n-m+1=4$, граф $\bar{\Gamma}$ имеет собственные значения 5 и - 4 . В этом случае $\bar{b}_{1}=18$ и $Г$ является дополнительным графом для графа из пунктов (2-3) заключения леммы 13.

Лемма 15. Если $b_{1}=21$, то выполняется одно из следующих утверждении:

(1) Г либо имеет параметры $(100,66,44,42),(136,30,8,6)$ или $(300,26,4,2)$, либо Г является псевдогеометрическим графом для $p G_{3}(9,3), p G_{9}(15,3), p G_{14}(20,3)$;

(2) Г является дополнительным графом для графа из пунктов $(1-2)$ заключения леммы 12 .

Доказательство. По предположению $m-1=3$ или 7 . Если $m-1=3$, то $n-m+1=7$, поэтому граф $\bar{\Gamma}$ имеет собственные значения 3 и -7 , поэтому $\bar{b}_{1}=24$. Теперь $k=24+\mu, \lambda=2+\mu, n=10$, по прямоугольному соотношению $\mu$ делит $24 \cdot 21$, а по условию целочисленности $10 \mu$ делит $3(24+\mu)(28+\mu)$. Поэтому 5 делит $(\mu-1)(\mu-2)$. Если $\mu=2$, то $\Gamma$ имеет параметры $(300,26,4,2)$. Если $\mu=6$, то $\Gamma$ имеет параметры $(136,30,8,6)$. Если $\mu=12$, то Г имеет параметры $(100,36,14,12)$ и является псевдогеометрическим графом для $p G_{3}(9,3)$. Если $\mu=36$, то Г имеет параметры $(96,60,38,36)$ и является псевдогеометрическим графом для $p G_{9}(15,3)$. Если $\mu=42$, то $Г$ имеет параметры $(100,66,44,42)$. Если $\mu=56$, то Г имеет параметры $(111,80,58,56)$ и является псевдогеометрическим графом для $p G_{14}(20,3)$.

Если $m-1=7$, то $n-m+1=3$, поэтому граф $\bar{\Gamma}$ имеет собственные значения 7 и -3. В этом случае $\bar{b}_{1}=16$ и Г является дополнительным графом для графа из пунктов $(1-2)$ заключения леммы 12 .

Лемма 16. Если $b_{1}=24$, то выполняется одно из следующих утверждений:

(1) Г имеет параметры $(196,39,14,6),(155,42,17,9)$ или $(120,77,52,44)$;

(2) Г является псевдогеометрическим графом для $p G_{3}(10,3), p G_{4}(11,3)$ или $p G_{14}(21,3)$;

(3) $Г$ является псевдогеометрическим графом для $p G_{\alpha}(5+\alpha, 4), \alpha \quad \epsilon$ $\{1, \ldots, 6,15,20,30,60\}$;

(4) Г является дополнительным графом $к$ одному из графов в пункте (1) заключения леммы 15 ;

(5) Г является дополнительным графом $к$ графу с параметрами $(121,30,11,6)$ или $(100,33,14,9)$.

Доказательство. По предположению $m-1=2,3,4,6$ или 8 . Если $m-1=2$, то $n-m+1=12$, поэтому $n=14$, граф $\bar{\Gamma}$ имеет собственные значения 2 и -12 , поэтому $\bar{b}_{1}=33$. Теперь $k=33+\mu, \lambda=8+\mu$, по прямоугольному соотношению $\mu$ делит $33 \cdot 24$, а по условию целочисленности $14 \mu$ делит $2(33+\mu)(36+\mu)$. Поэтому 7 делит $(\mu-2)(\mu+1)$. Если $\mu=2$, то Г имеет параметры $(456,35,10,2)$. Этот граф не существует ввиду границы Броувера-Ноймайера. Если $\mu=6$, то Г имеет параметры $(196,39,14,6)$ и является псевдогеометрическим графом для $p G_{2}(13,2)$. Если $\mu=9$, то Г имеет параметры $(155,42,17,9)$ и является псевдогеометрическим графом для $p G_{3}(14,2)$. Если $\mu=44$, то Г имеет параметры $(120,77,52,44)$. Последние три графа существуют. 
Если $m-1=3$, то $n-m+1=8$, поэтому граф $\bar{\Gamma}$ имеет собственные значения 3 и -8, поэтому $\bar{b}_{1}=28$. Теперь $k=28+\mu, \lambda=3+\mu, n=11$, по прямоугольному соотношению $\mu$ делит $28 \cdot 24$, а по условию целочисленности $11 \mu$ делит $3(28+\mu)(32+\mu)$. Поэтому 11 делит $(\mu-5)(\mu-1)$. Если $\mu=1$, то Г имеет параметры $(726,29,4,1)$, противоречие с тем, что 5 не делит 29 . Если $\mu=12$, то Г имеет параметры $(121,40,15,12)$ и является псевдогеометрическим графом для $p G_{3}(10,3)$. Если $\mu=16$, то Г имеет параметры $(111,44,19,16)$ и является псевдогеометрическим графом для $p G_{4}(11,3)$. Если $\mu=56$, то Г имеет параметры $(121,84,59,56)$ и является псевдогеометрическим графом для $p G_{14}(21,3)$.

Если $m-1=4$, то $n-m+1=6$, поэтому граф $\bar{\Gamma}$ имеет собственные значения 4 и -6 , поэтому $\bar{b}_{1}=25$. Теперь $k=25+\mu, \lambda=\mu, n=10$, по прямоугольному соотношению $\mu$ делит $25 \cdot 24$, а по условию целочисленности $10 \mu$ делит $4(25+\mu)(30+\mu)$. Поэтому 5 делит $\mu$. Если $\mu=5$, то Г имеет параметры $(175,30,5,5)$, это псевдо $G Q(6,4)$-граф. Если $\mu=10$, то $Г$ имеет параметры $(120,35,10,10)$ и является псевдогеометрическим графом для $p G_{3}(9,3)$. Если $\mu=15$, то $Г$ имеет параметры $(105,40,15,15)$ и является псевдогеометрическим графом для $p G_{9}(15,3)$. Если $\mu=20$, то Г имеет параметры $(100,45,20,20)$. Если $\mu=25$, то $Г$ имеет параметры $(99,50,25,25)$. Если $\mu=30$, то $Г$ имеет параметры $(100,55,30,30)$. Если $\mu=40$, то $Г$ имеет параметры $(105,65,40,40)$, противоречие с тем, что $v k$ нечетно. Если $\mu=50$, то $Г$ имеет параметры $(112,75,50,50)$. Если $\mu=60$, то $Г$ имеет параметры $(120,85,60,60)$. Если $\mu=75$, то $\Gamma$ имеет параметры $(133,100,75,75)$. Если $\mu=100$, то $Г$ имеет параметры $(156,125,100,100)$. Если $\mu=120$, то Г имеет параметры $(175,145,120,120)$, противоречие с тем, что $v k$ нечетно. Если $\mu=150$, то $\Gamma$ имеет параметры $(204,175,150,150)$. Если $\mu=200$, то Г имеет параметры $(253,225,200,200)$, противоречие с тем, что $v k$ нечетно. Если $\mu=300$, то Г имеет параметры $(352,325,300,300)$.

Если $m-1=6$, то $n-m+1=4$, поэтому $n=10$, граф $\bar{\Gamma}$ имеет собственные значения 6 и -4, поэтому $\bar{b}_{1}=21$. Поэтому Г является дополнительным графом к одному из графов в пункте (1) леммы 15.

Если $m-1=8$, то $n-m+1=3$, поэтому $n=11$, граф $\bar{\Gamma}$ имеет собственные значения 8 и -3 , поэтому $\bar{b}_{1}=18$. Поэтому $Г$ является дополнительным графом $\mathrm{k}$ графу с параметрами $(121,30,11,6)$ или $(100,33,14,9)$ (см. лемму 13$)$.

Лемма 17. Если $b_{1}=25$, то Г является дополнительным графом для одного из графов в пункте (3) заключения леммы 16.

Доказательство. По предположению $m-1=5, n-m+1=5$, поэтому $n=10$, граф $\bar{\Gamma}$ имеет собственные значения 5 и -5 , поэтому $\bar{b}_{1}=24$. Отсюда $\Gamma$ является дополнительным графом для одного из графов в пункте (3) заключения леммы 16. Лемма доказана.

Из лемм 3-17 следует теорема.

\section{Список литературы}

1. Brouwer A. E., Cohen A. M., Neumaier A., Distance-regular graphs. Springer-Verlag, Berlin, 1989.

2. Махнев А. А., О сильной регулярности некоторых реберно регулярных графов. Известия РАН, сер. матем. (2004) 68, 159-172.

3. Казарина В. И., Махнев А. А., О реберно регулярных графах с $b_{1}=5$. Владикавказский матем. журнал (2009) 11, №1, 29-42. 
4. Махнев А. А., О расширениях частичных геометрий, содержащих малые $\mu$-подграфы. Дискр. анализ и исслед. операчий (1996) 3, №3, 71-83.

5. Bussemaker F., Haemers W., Mathon R., Wilbrink H. A., A $(49,16,3,6)$ strongly regular graph does not exist. Europ. J. Comb. (1989) 10, 413-418.

6. Wilbrink H. A., Brouwer A. E., $(57,14,1)$ strongly regular graph does not exist. Proc. Kon. Nederl. Akad. Ser. A (1983) 45, 117-121.

7. Махнев А. А., О псевдогеометрических графах некоторых частичных геометрий. Вопросы алгебры (1997), № 11 60-67.

Статья поступила 21.05.2011. 